\title{
PERFORMANCE ENHANCEMENT OF WIND TURBINE POWER REGULATION BY SWITCHED LINEAR CONTROL
}

\author{
D.J.Leith \\ W.E.Leithead \\ Department of Electronic \& Electrical Engineering, \\ University of Strathclyde, \\ GLASGOW G1 1QE, U.K.
}

\begin{abstract}
Power regulation of horizontal-axis grid-connected up-wind constant-speed pitch-regulated wind turbines presents a demanding control problem with the plant, actuation system and control objectives all strongly nonlinear. In this paper a novel switched linear approach is devised. Conventional linear control and a nonlinear controller which, in some sense, optimises performance across the operating envelope provide benchmarks against which the switched control strategy is compared. In comparison with conventional linear control, the switched linear strategy reduces the peak power excursions experienced and the time spent at high power levels, with a consequent reduction in drive-train loads. It achieves very similar performance to the more complex nonlinear controller; that is, the performance is near optimal over the operational envelope. Moreover, in contrast to nonlinear control it admits straightforward, rigorous analysis and permits direct exploitation of the knowledge and experience accumulated with linear control. Hence, switched linear control is more suited for application to wind turbines than the nonlinear control strategy. The improvement in performance, in comparison to conventional linear control, is substantial.
\end{abstract}




\title{
PERFORMANCE ENHANCEMENT OF WIND TURBINE POWER REGULATION BY SWITCHED LINEAR CONTROL
}

\author{
D.J.Leith \\ W.E.Leithead \\ Department of Electronic \& Electrical Engineering,
University of Strathclyde
GLASGOW G1 1QE, U.K.
}

\section{Introduction}

Wind energy is one of the most promising sources of renewable energy for the U.K and over the last two decades there has been rapid development of wind turbine technology. The standard commercial design of turbine is a horizontal-axis grid-connected up-wind medium-scale machine with a rating of approximately $300 \mathrm{~kW}$ to $500 \mathrm{~kW}$. It is anticipated that the next generation of wind turbines which are presently being developed will include large-scale designs with a rating of around $1 \mathrm{MW}$. The rotor usually has two or three blades and in pitch regulated machines the pitch angle of either the full span of the blades, or just the outer tips, can be varied. The control design task for constant-speed pitch-regulated machines is to exploit this capability in order to regulate power output whilst minimising the load transients and thereby reducing fatigue damage. The objectives of the SISO control system are discussed fully by Leithead et al. (1991a, b, 1992).

Wind turbine power regulation presents a demanding SISO control problem with the plant, actuation system and control objectives all strongly nonlinear. Having catered adequately for the nonlinear plant dynamics (Leithead et al. 1991a, 1992, Leith \& Leithead 1995b), the plant may be considered to be essentially linear but the control objectives remain nonlinear. In this paper, a novel switched linear control strategy is presented which addresses the nonlinear control objectives. Its performance is compared to that of a nonlinear control strategy (Leith \& Leithead 1995a) which, in some sense, is optimised across the operating envelope rather than at an operating point. The latter, thereby, provides a benchmark against which other control strategies can be assessed.

In general, the field of switched linear control is presently immature (except for sliding mode control which has been the subject of specific interest for many years). One approach to the analysis of switched linear systems is to determine the global topology of trajectories in state-space (e.g. Banks \& Khathur 1989, Pettit \& Wellstead 1994). Unfortunately, this type of approach becomes intractable for high order systems. Morse \& Mayne (1992) note that stability is ensured if switching is sufficiently slow whilst Zhang (1993) extends a well-known stability result by Desoer (1969) for slowly time-varying systems to the discontinuous case. Employing Lyapunov methods, Becker et al. (1993) derive a sufficient condition for stability which takes the form of a smoothness requirement. However, these conditions are extremely restrictive in general and the development of methods which are not excessively conservative (ideally, non-conservative) remains unresolved. Nevertheless, switched linear control is commonly applied in a variety of situations. Its use to ensure the satisfaction of input and/or output constraints is widespread (e.g. Heise \& Maciejowski 1994, Campo et al. 1989), but largely ad hoc. Similarly, it is often used to satisfy changing performance objectives, e.g. in process control (Richalet 1993). While gain scheduled controllers may employ some form of interpolation of the control law between design operating points, simple switching is also used (e.g. Hyde \& Glover 1990). Switched linear controllers have been proposed for model-reference adaptive control schemes in order to improve robustness but this work is at a very early stage (Morse \& Mayne 1992, Morse 1993). In the present application, switched linear control is motivated by a pressing requirement for improved performance. Its use with performance enhancement as the primary objective has, unfortunately, received little previous attention (isolated work is reported by Gutman \& Hagander (1985) and Wredenhagen \& Belanger (1994), for example).

The paper is organised as follows. Section Two outlines the controller specification. In Section Three, the nonlinear controller of Leith \& Leithead (1995a) is briefly described and, in Section Four, the switched linear control approach is discussed, including the selection of an appropriate realisation. In Section Five, results from extensive simulations are used to compare the performance of these controllers with one another and with conventional linear control. Due to lack of space, attention is confined to continuous-time control of a typical $300 \mathrm{~kW}$ two-bladed machine with full-span pitch regulation. Similar results are obtained with other configurations of wind turbine (Leith \& Leithead 1994a,b). In Section Six, the conclusions are summarised. 


\section{Controller Specification}

In this paper, several controllers are described for a medium-scale wind turbine which is dynamically representative of commercial machines of its class. A block diagram of the linearised wind turbine control model is depicted in figure 1. The open-loop system dynamics, at a wind speed of $12 \mathrm{~m} / \mathrm{s}$, are modelled by the transfer function G(s) (see Leithead et al. 1991a for details of the nonlinear representation) where

$$
\mathrm{G}(\mathrm{s})=\frac{1.553 \times 10^{10}(\mathrm{~s}+4.0)}{\left(\mathrm{s}^{7}+1.073 \times 10^{2} \mathrm{~s}^{6}+5.917 \times 10^{3} \mathrm{~s}^{5}+2.263 \times 10^{5} \mathrm{~s}^{4}+4.393 \times 10^{6} \mathrm{~s}^{3}+3.927 \times 10^{7} \mathrm{~s}^{2}+1.932 \times 10^{8} \mathrm{~s}+4.423 \times 10^{8}\right)}
$$

It is important that fair comparisons between controller performance are made. To this end each controller investigated is required to have similar stability margins and to operate within the same actuator restrictions. All the controllers are designed to meet the following requirements :

(i) Gain margin of at least $10 \mathrm{~dB}$.

(ii) Phase margin of approximately 60 degrees.

(iii) Servo pitch acceleration standard deviation no more than approximately $20 \mathrm{deg} / \mathrm{s}^{2}$.

The aerodynamic behaviour of wind turbine blades is highly nonlinear and strongly dependent on wind speed. In terms of a linearised plant description, as wind speed increases the gain of the plant increases since the rate of change of aerodynamic torque to pitch angle increases. It is standard practice for wind turbine controllers to include a nonlinear gain to compensate for this variation and make the control task essentially linear (Leith \& Leithead 1995b, Leithead et al.1991a). Because of their global mutual compensation (Leith \& Leithead 1995b), these two nonlinearities are ignored in the remainder of this paper. (The dynamics at all wind speeds may now be considered to be the same and modelled by G(s)). However, the representation of the aerodynamics is very basic and subject to considerable uncertainty. Consequently, a good gain margin, in conjunction with a good phase margin, is particularly important in order to achieve adequate stability margins. Because of the complexity of the interaction of the rotor with the wind, it is not possible to quantify the uncertainty in the aerodynamic gain but practical experience indicates that $10 \mathrm{~dB}$ is an appropriate gain margin. If adequate gain and phase margins are not achieved the system must sometimes destabilise, although not necessarily become unstable, in which case the wind turbine would experience large load fluctuations.

Requirement (iii) represents a practical limitation on the level of activity of the blade servo. Servo pitch acceleration is a measure of the force or torque developed by the actuator and the standard deviation reflects activity over the medium and long term. It should be emphasised that the value of pitch acceleration used is not that of the actual turbine blades. Rather, it is a normalised measure which permits valid comparisons to be made between differing designs of actuator. For example, blade pitching systems with different gearing ratios linking the actuator to the blades may be compared in an unbiased manner using this measure. The pitch acceleration of the actual blades will typically have a lower value as a result of the compliance of the blades and linkages together with many other factors. The restriction on actuator activity inherent in (iii) is always necessary to prevent saturation occurring too frequently but there may also be additional hardware reasons for its imposition. For the machine considered here, the actuator is an electro-mechanical system and the restriction on servo pitch acceleration is equivalent to a restriction on the servo motor current which is imposed to prevent over-heating. The limit of $20 \mathrm{deg} / \mathrm{s}^{2}$ is typical of comparable commercial machines.

There are several implementation issues which need to be considered. The actuator, in addition to requirement (iii), is subject to hard limits on torque, velocity and position and effective anti-wind-up measures are therefore important. There is, also, the requirement to ensure smooth and timely start-up of the controller. (When the wind speed falls below a certain level, rated power cannot be generated and control action is suspended until the wind speed rises again). These issues have a significant impact on performance and are discussed in detail elsewhere (Leith \& Leithead 1995b, Leithead et al. 1991a). It should be noted that the nature of the realisation of the controller is important when addressing the implementation issues and also impacts strongly upon the effectiveness of the compensating nonlinear gain discussed above. The controller realisations adopted in this paper are compatible with the requirements thereby imposed (Leith \& Leithead 1995b, Leithead et al.1991a).

\section{Nonlinear Control}


The actuator characteristics, especially the limits on torque, are one of the main restrictions on the performance that can be achieved by a controller. As the wind speed rises, a linear controller places less demand on the actuator since the sensitivity of the aerodynamic torque to pitch changes increases faster than the sensitivity to wind speed changes. Hence, for a controller with fixed open-loop cross-over frequency, while the actuator may be worked to its full capability at low wind speed, it may not be used as fully at higher wind speeds. However, it is at these higher wind speeds that loads are greatest and therefore controller performance is most critical. Parametric studies (Rogers \& Leithead 1993, 1994 and Leithead and Rogers 1993) indicate that there is an advantage in using this spare actuator capacity as the wind speed rises and that there exists an optimum level of activity for the controller at each wind speed. Whether, at any particular wind speed, the resulting optimum cross-over frequency can be achieved in practice depends on the capabilities of the actuator.

The optimum, as measured by the standard deviation of the power transients, see figure 2, exists due to the action of two competing factors. As wind speed rises, for a fixed controller the standard deviation of the power also rises due to the increased level of turbulence. It is therefore attractive to increase the controller activity by raising the open-loop cross-over frequency, giving improved disturbance rejection. However, the wind spectrum differs from that experienced at a static point (see for example Leithead et al. 1991a). In particular, the wind experienced by a wind turbine contains large amounts of energy at frequencies $\mathrm{nP}$, where $\mathrm{n}$ is the number of blades or an integer multiple thereof, and $\mathrm{P}$ is the rotational speed of the rotor. Since it is necessary to protect the actuator by causing the open-loop transmittance to roll-off, whilst maintaining adequate gain and phase margins, there is an inevitable tendency for the sensitivity function to increase the intensity of the nP peaks as the cross-over frequency is increased.

The requirement is to design a controller which operates as near as possible to its optimal level of activity in all wind speeds, subject to actuator constraints. A complication is the lack of a direct measurement of wind speed. Indeed there is no such thing as 'the windspeed' experienced by a wind turbine, since the rotor experiences a spatially and temporally distributed wind field. Simple scheduling is therefore not appropriate and the wind speed must be inferred from the plant dynamics via the pitch demand. If the controller is operating correctly, the demanded pitch angle is a good indicator of wind speed. (This approach is widely used to vary the previously noted nonlinear gain, which essentially linearises the plant by compensating for variations in the aerodynamic torque sensitivity). Employing an internal state of the system, such as the pitch demand, to implicitly change the controller as wind speed varies must be treated with some caution, however, since it introduces additional nonlinear feedback loops, thereby changing the plant dynamics. The design task is to develop a continuously varying controller which induces the appropriate closed-loop dynamics at any wind speed, despite the presence of these feedback loops. A nonlinear control strategy which achieves this objective is discussed in detail in Leith \& Leithead (1995a). Briefly, a family of linear controllers is designed at various wind speeds using classical loop-shaping design techniques. Some care is required to minimise the differences between these controllers so that interpolation between them can be carried out as smoothly as possible. The continuous family of controllers thereby generated is :

$$
\begin{aligned}
& \left(s^{2}+7.59 s+68.06\right) \quad(s+1.7)(s+1.8)\left(s^{2}+3 s+416.16\right) \\
& \mathrm{g}=\frac{\left(\mathrm{s}^{2}+\mathrm{as}+\mathrm{b}\right) \quad \mathrm{s}(\mathrm{s}+0.3)(\mathrm{s}+3.75)\left(\mathrm{s}^{2}+8 \mathrm{~s}+416.16\right)}{(\mathrm{s}+1.7)(s+1.8)\left(s^{2}+3 s+446.16\right)} \\
& \left(s^{2}+2 s+104.04\right)\left(s^{2}+7.243 s+38.637\right) 2209 \\
& \mathrm{x}-\left(\mathrm{s}^{2}+11 \mathrm{~s}+104.04\right)(\mathrm{s}+100)(\mathrm{s}+30)\left(\mathrm{s}^{2}+65.8 \mathrm{~s}+2209\right)
\end{aligned}
$$

where

$$
\begin{aligned}
& a=-0.033047 p^{2}+0.75064 p+3.3749 \\
& b=2.6002 p+58.040 \\
& g=(0.13779 p+0.29784)
\end{aligned}
$$

and $\mathrm{p}$ is the pitch angle demanded by the controller, in degrees. It can be seen that these controller transfer functions are the same except for a varying gain and a pair of varying poles. The Bode plot of the open-loop transfer function of the system with the member of this family of controllers for $12 \mathrm{~m} / \mathrm{s}$ wind speed is shown in figure 3 . The family of controllers has the following features : 
- Low frequency shaping to improve disturbance rejection.

- Notches at 2P and $4 \mathrm{P}$ to reduce actuator activity and reduce the enhancement of the loads induced by these spectral peaks.

- High frequency roll-off to reduce actuator activity.

Upper and lower bounds are placed on a, b and g,. When p is less than 3.84 degrees (corresponding to $12 \mathrm{~m} / \mathrm{s}$ wind speed), a, b and $\mathrm{g}$ are held at their 3.84 degree values. Similarly, when $\mathrm{p}$ is greater than 20.59 degrees $(24 \mathrm{~m} / \mathrm{s}$ wind speed), a, b and $\mathrm{g}$ are held at their 20.59 degree values.

The controller is split into two main blocks as shown in figure 4 to cater for the situation when a negative pitch angle is demanded, i.e. when the wind speed has fallen below the level at which rated power of $300 \mathrm{~kW}$ can be generated. In this situation the controller is switched out of operation. Due to the presence of low frequency dynamics within the controller, transients may occur for a substantial period of time when the controller is switched back in again as the wind speed rises. To combat the transients, a minor loop is introduced within the controller which is activated during below rated operation so that the controller is continuously operating and thereby smooth switching achieved. This technique, including the partitioning of the controller into inner and outer blocks, is discussed in detail in Leith \& Leithead (1995b) and Leithead $e t$ al. (1991a, 1992). The controller partitioning in the present case is as follows:

\section{Inner Block}

$$
\text { g 4.5 } \frac{(\mathrm{s}+1.7)(\mathrm{s}+1.8)\left(\mathrm{s}^{2}+7.59 \mathrm{~s}+68.06\right)}{\mathrm{s}(\mathrm{s}+0.3)(\mathrm{s}+3.7)\left(\mathrm{s}^{2}+\mathrm{as}+\mathrm{b}\right)}
$$

\section{Outer Block}

$$
0.222 \frac{\left(s^{2}+7.243 s+38.637\right)\left(s^{2}+2 s+104.04\right)}{(s+100)(s+30)\left(s^{2}+11 s+104.04\right)\left(s^{2}+65.8 s+2209\right)}
$$

The realisation adopted for the inner block is that of figure 5 (Leith \& Leithead 1995a).

\section{Switched Linear Control}

In the preceding section, a nonlinear controller is discussed which not only utilises the maximum actuator capability at all wind speeds but is instantaneously the most appropriate controller at all wind speeds. In some sense, the nonlinear controller may be considered to be optimised across the operating envelope rather than at an operating point. It is noted by Leith \& Leithead (1995a) that whilst stability margins are not established in a completely rigorous manner the optimisation pertains to both local and non-local behaviour. It thereby constitutes a benchmark against which other control strategies can be assessed. In this section, a dual-mode type of controller is designed which switches between two linear controllers, one suitable for low wind speeds and the other suitable for high wind speeds. As discussed previously, no measurement of wind speed is possible and an internal state of the controller, namely pitch demand, is used to determine switching. The result is a fast switched linear system. Owing to the rapid variation in pitch demand that typically occurs, it cannot be assumed that switching will be slow; that is, there may be only very brief intervals between switch transitions. In comparison to the nonlinear controller of section three, the switched controller is anticipated to have poorer performance both because it does not continuously adjust with wind speed and because switching can induce large transients (as is the case when switching from below to above rated operation, Leithead et al. 1991a, Leith \& Leithead 1995b). However, it is expected that the design may be simpler and that stability margins might be more firmly established.

As mentioned in the preceding section, the demand which a linear controller places on an actuator decreases with rising wind speed. It does so rapidly in lower wind speeds below $15 \mathrm{~m} / \mathrm{s}$ but much less 
rapidly in higher wind speeds above $18 \mathrm{~m} / \mathrm{s}$. However, it is at these higher wind speeds that controller performance is most critical. These considerations suggest than the appropriate wind speed at which to switch between the two controllers lies between $15 \mathrm{~m} / \mathrm{s}$ and $18 \mathrm{~m} / \mathrm{s}$. A wind speed of $16 \mathrm{~m} / \mathrm{s}$ (corresponding to 11.14 degrees pitch demand) is selected as the switching point. A conventional design of linear controller is used in wind speeds below this threshold with a more active linear controller at higher wind speeds. These controllers have the following transfer functions :

\section{Low-Wind Speed Controller}

$$
\begin{aligned}
& 871.229 \frac{(\mathrm{s}+1.6)^{2}\left(\mathrm{~s}^{2}+7.243 \mathrm{~s}+38.637\right)}{\mathrm{s}(\mathrm{s}+0.3)(\mathrm{s}+3.7)(\mathrm{s}+20)(\mathrm{s}+50)} \\
& \mathrm{x} \frac{\left(\mathrm{s}^{2}+1.5 \mathrm{~s}+104.04\right)\left(\mathrm{s}^{2}+6 \mathrm{~s}+416.16\right)}{\left(\mathrm{s}^{2}+11 \mathrm{~s}+104.04\right)\left(\mathrm{s}^{2}+10 \mathrm{~s}+416.16\right)\left(\mathrm{s}^{2}+65.8 \mathrm{~s}+2209\right)}
\end{aligned}
$$

(gain margin $10 \mathrm{~dB}$, phase margin 56.14 degrees, open-loop cross-over frequency $1.826 \mathrm{r} / \mathrm{s}$ ). The low wind speed controller is similar to previous controllers used with a commercial two-bladed design of wind turbine (Leithead \& Agius 1991, Bossanyi et al. 1992).

$\underline{\text { High-Wind Speed Controller }}$

$$
5591.91 \frac{(\mathrm{s}+1.7)(\mathrm{s}+1.8)\left(\mathrm{s}^{2}+3 \mathrm{~s}+416.16\right)\left(\mathrm{s}^{2}+7.59 \mathrm{~s}+68.06\right)}{\mathrm{s}(\mathrm{s}+0.3)(\mathrm{s}+3.7)\left(\mathrm{s}^{2}+8 \mathrm{~s}+416.16\right)\left(\mathrm{s}^{2}+14.67 \mathrm{~s}+100\right)}
$$

(gain margin $10 \mathrm{~dB}$, phase margin 55.62 degrees, open-loop cross-over frequency $2.85 \mathrm{r} / \mathrm{s}$ ). The high wind speed controller is the member for a wind speed of $20 \mathrm{~m} / \mathrm{s}$ (16.21 degrees pitch) of the family of controllers described in section three. With these choices of controller and switching threshold, the open-loop crossover frequency of the switched system as a function of wind speed is, in some sense, a best fit to the crossover frequency of the nonlinear controller of section three as a function of wind speed (see figure 6).

A switched linear system employing linear elements which individually are closed-loop unstable can be stabilised for appropriate switching (see, for example, Peleties \& DeCarlo 1993). However, the class of admissible switching rules is greatly restricted in comparison to switched linear systems for which the closedloop system is stable when each of the linear controllers in the switched controller is used individually. In particular, the rule employed in the present application is inadmissible if the linear elements are unstable since in a steady wind the pitch demand is constant and one of the individual controllers is in continuous use; that is, the closed-loop system is unstable. Hence, it is assumed in the following that the linear component systems are stable. Employing Lyapunov methods, Becker et al. (1993) derive a sufficient condition for stability which is applicable to switched linear systems (including rapid switching). The condition requires that a single function exists which acts as a Lyapunov function for every member of the family of linear systems employed in the switched system. It may be interpreted as a smoothness condition which holds if the members of the linear family are sufficiently alike (trivially, when they are identical). That sufficient smoothness (in some sense) ensures stability is intuitively reasonable. However, the Lyapunov condition of Becker et al. (1993) is extremely restrictive in general, and therefore of limited utility in practice. Shevitz \& Paden (1994) comment that it is natural to consider nonsmooth Lyapunov function when analysing nonsmooth systems and extend Lyapunov theory to include a class of such functions. The result of Becker et al. (1993) could, therefore, be generalised to lessen its conservativeness, but such an approach is of limited use in practice owing to the difficulty of determining suitable nonsmooth Lyapunov functions and the tractability of the result of Becker et al. (1993) is lost.

A more practical test for stability of switched linear systems than those discussed above is required. The Small Gain theorem (Desoer \& Vidyasagar 1975) can be employed to provide a suitable sufficiency 
condition for $L_{2}$ stability of the switched control law, as follows. Rearranging the controlled system as shown in figure 7 and taking $\Gamma \equiv 1$, the transfer function between $x$ and $y$ is $-\left(G_{2}-G_{1}\right) /\left(1+G_{1}\right)$. The system now consists of a SISO linear time-invariant block with nonlinear feedback provided by the switch and it is in a form suitable for the use of the Small Gain theorem and criteria derived from it, such as the Circle Criterion (see, for example, Desoer \& Vidyasagar 1975). The switch is an operator with induced $L_{2}$ norm in the range $(0,1]$; therefore, by direct application of the Small Gain theorem a sufficient condition for closed-loop stability of the switched system is that the magnitude of the transfer function $-\left(G_{2}-G_{1}\right) /\left(1+G_{1}\right)$ should not exceed unity. Again, the condition may be interpreted as a smoothness condition, which is satisfied if the difference between the system transfer functions, $\mathrm{G}_{1}$ and $\mathrm{G}_{2}$, for the switched controller is sufficiently small. In addition to individually satisfying the appropriate performance and stability requirements, the linear elements should be jointly designed to shape the transfer function $\left(G_{2}-G_{1}\right) /\left(1+G_{1}\right)$ in such a way as to satisfy this smoothness condition (or, similarly, one employing the Circle Criterion). In the present application, $\mathrm{G}_{1}$ contains an integrator term, and $1 /\left(1+G_{1}\right)$ therefore tends to zero at low frequencies. In addition, $G_{1}$ is strictly proper, and so $1 /\left(1+\mathrm{G}_{1}\right)$ tends to unity at high frequencies. Hence, the admissible difference between $\mathrm{G}_{1}$ and $\mathrm{G}_{2}$ may be large at low frequencies but is required to be small at high frequencies (that is, less than unity). A plot of the magnitude of $\left(\mathrm{G}_{2}-\mathrm{G}_{1}\right) /\left(1+\mathrm{G}_{1}\right)$ for the present switched system is shown in figure 8 . It can be seen from this plot that the magnitude less than unity at all frequencies and hence the switched system is stable; that is, the linear elements selected satisfy the smoothness test immediately and do not require further modification to shape $\left(\mathrm{G}_{2}-\mathrm{G}_{1}\right) /\left(1+\mathrm{G}_{1}\right)$. Notice, however, that this transfer function exhibits peaks at frequencies in the vicinity of the cross-over frequencies of the linear elements. Hence, shaping of $\left(\mathrm{G}_{2^{-}}\right.$ $\left.\mathrm{G}_{1}\right) /\left(1+\mathrm{G}_{1}\right)$ can be expected, in general, to have a significant influence upon the design of the linear elements.

Assessment of stability robustness is also necessary. As mentioned in section two, the dominant source of uncertainty typically lies in the aerodynamic behaviour of the turbine blades which may be represented by $\Gamma \neq 1$ in figure 7 . Robustness to variations in $\Gamma$ is assessed rigorously using a small gain approach once again. The system now contains two nonlinear terms, $\Gamma$ and the switch. Straightforward reformulation of the closed-loop system so that these nonlinearities lie in the feedback path gives rise to the following 2-input/2output transfer function matrix in the forward path:

$$
\left[\begin{array}{l}
\mathrm{x} \\
\mathrm{e}
\end{array}\right]=\left[\begin{array}{cc}
\mathrm{G}_{2}-\mathrm{G}_{1} & 0 \\
-\mathrm{G}_{1} & -1
\end{array}\right]\left[\begin{array}{l}
\mathrm{z} \\
\mathrm{y}
\end{array}\right]
$$

The induced $L_{2}$ norm of this transfer function is $\sup \sigma_{\max }(\omega)$, where $\sigma_{\max }(\omega)$ is the maximum singular value of the transfer function matrix at frequency $\omega$ and the supremum is over all frequencies in the range $[0, \infty)$ (Desoer \& Vidyasagar 1975). By the Circle Criterion, the system is determined to be stable for $|\Gamma|_{2}$ in the range $(0.81,2.19)$ which compares to a $|\Gamma|_{2}$ range of $(0.38,2.63)$, with the low windspeed controller on its own and $(0.64,2.27)$ with the high windspeed controller.

Of course, the rigour of this approach is counter-balanced by its inherent conservativeness. In particular, the robustness to variation in the magnitude of the gain is insufficient to meet the performance requirements. Consequently, the stability margins derived are too conservative and it is important that less conservative results are obtained. This may be achieved using harmonic balance techniques (see, for example, Atherton 1982) at the price of reduced rigour. The uncertainty in the aerodynamic behaviour of the turbine blades may be considered largely as a gain uncertainty. Since the describing function of a real, memoryless, timeinvariant nonlinear gain is real, the gain margin is indicated as a useful measure of robustness. The gain margin may be assessed by perturbing the value of $\Gamma$ and evaluating the effect on the closed-loop stability. The switched system is determined to possess a gain margin of $10.0 \mathrm{~dB}$. By similar means, the phase margin is determined to be approximately 55 degrees; that is, the stability margins of the component linear systems are retained by the switched system. Simulation results confirm these gain and phase margins are not conservative for this application.

The above analysis, while indicating that the switched system meets the requirement for robust stability, does not preclude the possibility that large transients might be generated by the frequently occurring switching between controllers. The source of the transients would be the discontinuities which switching introduces into signals within the controller, particularly as the controller transfer functions both contain an integrator and a low frequency pole (required in order to achieve adequate low frequency disturbance rejection) which prolong their presence. The performance of the switched system may well not be acceptable if large transients are present and the requirement for smooth switching is essential. It is noted in Leith \& Leithead (1995a) that selecting an appropriate realisation is an important part in the design of nonlinear controllers. This is also the case for the switched controller and the requirement for smooth switching may 
be met by adopting the realisation depicted in figure 9. While only one controller acts on the plant at any time, it is ensured that the high and low wind speed controllers are both continuously driven by the same input. Initialisation transients associated with the low frequency controller dynamics are thereby avoided. In addition, the pure integration term, which is common to both the high and low wind speed controllers, is located after the other controller dynamics with the switch positioned immediately prior to it. Owing to the switch, the input to the integrator may be discontinuous. However, for an input with finite magnitude (that is, the system is stable), the output of the integrator is continuous. Extensive simulations confirm that these simple techniques are extremely effective in preventing unacceptable switching transients. Moreover, the integrator acts to smooth any chatter of the switch. The realisation adopted therefore provides an elegant alternative to the more conventional solution of introducing hysteresis. Minor loops to handle the transition from below to above rated power operation are also depicted in figure 9. These loops are similar to the minor loop noted in section three for the nonlinear controller and are discussed in detail in Leith \& Leithead (1995b) and Leithead et al. (1991a, 1992). Note that a minor loop is required for each controller because the switching when $\mathrm{u}$ equals 0 degrees (below to above rated transition) and when $\mathrm{u}$ equals 11.14 degrees (controller transition) may occur in close proximity. However, the integrator can be included in the minor feedback loop for the high wind speed controller as the timing is less critical for this controller. The controller inner/outer block partitioning in the present case are as follows:

Inner Block 1

$$
\frac{(s+1.6)^{2}}{s(s+0.3)(s+3.7)}
$$

\section{Outer Block 1}

390.993

$$
\left(s^{2}+7.243 s+38.637\right)\left(s^{2}+1.5 s+104.04\right)\left(s^{2}+6 s+416.16\right)
$$

$$
(s+20)(s+50)\left(s^{2}+11 s+104.04\right)\left(s^{2}+10 s+416.16\right)\left(s^{2}+65.8 s+2209\right)
$$

\section{Inner Block 2}

$$
(\mathrm{s}+1.7)(\mathrm{s}+1.8)\left(\mathrm{s}^{2}+7.59 \mathrm{~s}+68.06\right)
$$

$$
s(s+0.3)(s+3.7)\left(s^{2}+a s+b\right)
$$

\section{Outer Block 2}

1218.484

$$
\left(s^{2}+7.243 s+38.637\right)\left(s^{2}+2 s+104.04\right)\left(s^{2}+3 s+416.16\right)
$$

$$
(s+100)(s+30)\left(s^{2}+11 s+104.04\right)\left(s^{2}+65.8 s+2209\right)\left(s^{2}+8 s+416.16\right)
$$

\section{Performance Comparison}

The performance of the nonlinear and novel switched linear control strategies is investigated using a well validated simulation methodology. For comparison, a conventional linear controller designed to meet the same specifications is also considered:

Linear Controller (see for example Leithead et al. 1991a, 1992):

$$
871.229 \frac{(\mathrm{s}+1.6)^{2}\left(\mathrm{~s}^{2}+7.243 \mathrm{~s}+38.637\right)}{\mathrm{s}(\mathrm{s}+0.3)(\mathrm{s}+3.7)(\mathrm{s}+20)(\mathrm{s}+50)}
$$




$$
x \frac{\left(s^{2}+1.5 s+104.04\right)\left(s^{2}+6 s+416.16\right)}{\left(s^{2}+11 s+104.04\right)\left(s^{2}+10 s+416.16\right)\left(s^{2}+65.8 s+2209\right)}
$$

(gain margin $10 \mathrm{~dB}$, phase margin 56.14 degrees, cross-over frequency $1.826 \mathrm{r} / \mathrm{s}$ ). This controller is similar to previous controllers used with a commercial two-bladed design of wind turbine (Leithead \& Agius 1991, Bossanyi et al. 1992), and corresponds to the low wind speed component of the switched linear control strategy discussed in section four.

Assessment of performance follows the well-validated approach of Leithead \& Agius (1991) and Bossanyi et al. (1992). Simulation runs are performed with each specific controller over a range of wind speeds and turbulence levels. Four mean wind speeds of 12, 16, 20 and $24 \mathrm{~m} / \mathrm{s}$ were used at three nominal turbulence levels of 10, 15 and $20 \%$. Each simulation run is of 260 seconds duration, giving 4 one minute periods of data per run, after discarding the initial 20 seconds to allow the system to settle down, and 48 one minute periods over all the runs. A data sampling rate of $50 \mathrm{~Hz}$ is used. The nominal turbulence level only applies over a long time period and the range of turbulence levels for the one minute samples is $6-26 \%$. While turbulence in the range $8-18 \%$ corresponds to the moderate wind conditions noted Leithead \& Agius (1991) and Bossanyi et al. (1992), results for slightly more turbulent conditions with intensity in the range 13 - $26 \%$ are presented in this paper. For each one minute sample, within the specified turbulence range, the maximum power was plotted against the mean wind speed over that sample. A linear fit to this data then provides an indication of the trend in maximum power with wind speed. Moreover, if the standard deviation of the residues of the maxima about the linear fit is determined, then the power maxima experienced under normal operating conditions are unlikely to exceed the linear fit by more than three times the standard deviation. Empirical investigations (Leithead \& Agius 1991, Bossanyi et al. 1992) have shown that despite the small number of data points used, this approach is nevertheless a good indicator of the comparative performance between controllers.

Before considering results based on the above one minute binning approach, an indication of the relative performance of the controllers is obtained by comparing the probability distributions of the power time histories for these controllers. These are given in figure 10 for typical power time histories at a mean wind speed of $24 \mathrm{~m} / \mathrm{s}, 20 \%$ turbulence intensity. A large reduction in the time spent at high power levels is evident with both the switched and nonlinear controllers which can be seen to have very similar performance. For example, the percentage of time that the power level exceeds $450 \mathrm{~kW}$ for the various controllers is as follows

\begin{tabular}{|c|c|}
\hline Controller & $\begin{array}{c}\text { Exceedance } \\
\text { Probability } \\
450 \mathrm{~kW}\end{array}$ \\
\hline linear & $4.02 \%$ \\
switched & $0.62 \%$ \\
nonlinear & $0.64 \%$ \\
\hline
\end{tabular}

Similar results are obtained at other wind speeds and turbulence levels.

The equations of linear fits to the power maxima, from the one minute samples with turbulence in the range $13-26 \%$, are given in the table below. The plot in figure 11 shows the three standard deviation line associated with each fit. As noted, this line provides an indication of the maximum power likely to be encountered during normal operation. Once again, the performance of the switched and nonlinear controllers is extremely similar and a substantial improvement over linear control.

\begin{tabular}{|c|c|c|}
\hline Controller & Fit & Standard Deviation \\
\hline linear & $8.49 \mathrm{w}+280.05$ & 20.02 \\
switched & $5.79 \mathrm{w}+317.17$ & 14.19 \\
nonlinear & $5.11 \mathrm{w}+330.49$ & 14.52 \\
\hline
\end{tabular}

The linear controller's maxima increase at the fastest rate while the switched and nonlinear controllers increase at almost two-thirds this rate. The reduced rate of increase of the maxima in combination with much lower standard deviations of the residues, corresponding to tighter bunching of the maxima, represents a significant overall reduction in the peak power excursions likely to be experienced and a consequent reduction in drive-train load transients. 
The pitch acceleration standard deviations for the one minute samples are shown in figure 12. The linear controller works the actuator hardest at low wind speeds but the actuator activity falls rapidly as the wind speed rises due to the increase in the sensitivity of the aerodynamic torque to pitch changes. In contrast, the standard deviation for the switched and nonlinear controllers remains roughly constant as wind speed rises, exploiting the extra actuator capacity available at higher wind speed as intended.

More detailed results are contained in Leith \& Leithead (1994a,b) together with results for a three-bladed machine.

\section{Conclusion}

In the control design task for pitch-regulated constant-speed wind turbines, the plant, actuation system and control objectives are all strongly nonlinear. Having catered adequately for the nonlinear plant dynamics (Leithead et al. 1991a, 1992, Leith \& Leithead 1995b), the plant may be considered to be essentially linear but the control objectives remain nonlinear. Improvement in the controller performance can still be achieved by adjusting the controller as the operating point changes. Wind speed fluctuations are highly stochastic and the operating point of a wind turbine varies rapidly and continuously over the whole operational envelope. Whilst, typically, the bandwidth of the closed-loop system is $3 \mathrm{r} / \mathrm{s}$, the operating point might cover its full range in one or two seconds. Consequently, the emphasis is on the nonlinear behaviour and performance when considering control strategies which are adjusted as the operating point changes.

In the control strategy of Leith \& Leithead (1995a) the control algorithm continuously changes with the operating point in such a way that the controller is instantaneously always the most appropriate for the inferred wind speed. The result is a nonlinear control strategy which, in some sense, may be considered to be optimised across the operating envelope rather than at an operating point. Although the establishment of stability margins is not entirely satisfactory, the optimisation pertains to both local and non-local behaviour of the controller. It, thereby, provides a benchmark against which the other control strategies can be assessed.

In this paper, a switched linear control strategy is presented which is novel both in terms of the application and the motivation: namely, a pressing requirement for improved performance. The controller consists of two control algorithms, one appropriate for low wind speeds the other appropriate for high wind speeds. The resulting switched linear control strategy is anticipated to perform less well than the continuously varying nonlinear strategy but offers several advantages. The Small Gain theorem is employed to establish a sufficient condition which ensures the stability of switched linear systems and supports, in a straightforward manner, the rigorous analysis of the switched linear system, with no need for approximations and assumptions. In particular, stability margins can be firmly established. Since the stability condition is based in the frequency domain it facilitates straightforward and direct 'tuning' of the controller design. The switched linear strategy permits direct exploitation of existing knowledge and experience of linear control. Furthermore, design and implementation of the switched linear control algorithm is typically more straightforward than that of the continuous nonlinear algorithm.

From the results of extensive runs using a well validated simulation methodology, the performance of a typical two-bladed configuration of wind turbine is compared for the nonlinear controller, the switched linear controller and a conventional linear controller. The simulations confirm that the switched controller performs as intended. It is found to reduce both the peak power and the time spent at high power levels in comparison to linear control, with a consequent reduction in drive-train loads. The improvement is obtained by exploiting the actuator capability left unused at higher wind speeds by linear time-invariant controllers and is cheap in the sense that to achieve the same improvement by means of linear control, were it possible within practical constraints, would require much greater and expensive actuator capability than is typically available. Contrary to expectation, the switched linear control strategy achieves very similar performance to the nonlinear control strategy; that is, the performance is near optimal over the operational envelope. Hence, because of the previously mentioned advantages, the switched linear control strategy is more suited for application to wind turbines than the nonlinear strategy. The improvement in performance, in comparison to conventional linear control, is substantial.

\section{Acknowledgements}

The EPSRC, DTI (formerly D.En) and ETSU, by whose permission this paper is published, are gratefully acknowledged for supporting the work presented. 


\section{References}

ATHERTON, DP, 1982, Nonlinear Control Engineering (New York: Van Nostrand Reinhold).

BANKS, S.P., KHATHUR, S.A., 1989, Structure and Control of Piecewise-Linear Systems. International Journal of Control, 50, 667-686.

BECKER, G., PACKARD, A., PHILBRICK, D., BALAS, G., 1993, Control of Parametrically-Dependent Linear Systems: A Single Quadratic Lyapunov Approach. Proceedings of the American Control Conference, San Francisco, 2795-2799.

BOSSANYI, E.A., SMITH, G.J., LEITHEAD, W.E., AGIUS, P., 1992, Design and Testing of a Classical Controller for the MS-3. Wind Turbine Final Report E/5A/CON/6033/2184, Energy Technology Support Unit, Harwell, Oxfordshire OX11 ORA, U.K.

CAMPO, P.J., MORARI, M., NETT, C., 1989, Multivariable Anti-Windup \& Bumpless Transfer: A General Theory. Proceedings of the American Control Conference, Pittsburgh, 1706-1711.

DESOER, C.A., 1969, Slowly Varying System dx/dt=A(t)x. IEEE Transactions on Automatic Control, 14, 780-781.

DESOER, C.A., VIDYASAGAR, M.,1975, Feedback Systems: Input-Output Properties (London : Academic Press).

GUTMAN, P., HAGANDER, P., 1985, A New Design of Constrained Controllers for Linear Systems. IEEE Transactions on Automatic Control, 30, 22-33.

HEISE, S.A., MACIEJOWSKI, J.M., 1994, Stability of Constrained MBPC using an internal model control structure. pp230-244 in Advances in Model-Based Predictive Control edited by D.Clarke (New York: Oxford University Press).

HYDE, R.A., GLOVER, K., 1990, VSTOL Aircraft Flight Control System Design using $\mathrm{H}_{\infty}$ controllers and a switching strategy. Proceedings of the 29th Conference on Decision and Control, Honolulu.

LEITH, D.J., LEITHEAD, W.E., 1994a, Comparison of Various Control Strategies for a Two-Bladed Wind Turbine. Proceedings of the British Wind Energy Association Conference, Stirling.

LEITH, D.J., LEITHEAD, W.E., 1994b, Benefits of Optimising the Controller with Windspeed for a Constant Speed HAWT. Proceedings of the European Wind Energy Conference, Greece.

LEITH, D.J., LEITHEAD, W.E., 1994c, Application of Nonlinear Control to a HAWT. Proceedings of the 3rd IEEE Conference on Control Applications, Glasgow.

LEITH, D.J., LEITHEAD, W.E., 1995a, Appropriate Realisation of Gain-Scheduled Controllers with Application to Wind Turbine Regulation. Submitted to International Journal of Control.

LEITH, D.J., LEITHEAD, W.E., 1995b, An Investigation of the Benefits of Nonlinear Control for PitchRegulated Wind Turbines. Report under DTI Agreement No. ETSU W/42/00348/00/00, Industrial Control Centre, Department of Electronic \& Electrical Engineering, University of Strathclyde, Glasgow.

LEITHEAD, W.E., AGIUS, P., 1991, Application of Classical Control to the WEG MS3 Wind Turbine. Report ICU/342, Industrial Control Centre, Department of Electronic \& Electrical Engineering, University of Strathclyde, Glasgow.

LEITHEAD, W.E., DE LA SALlE, S.A., REARDON, D.L., GRIMBLE, M.J., 1991a, Wind Turbine Control Systems Modelling and Design Phase I and II. Department of Trade \& Industry Report No. ETSU WN 5108.

LEITHEAD, W.E., DE LA SALLE, S.A., REARDON, D., 1991b, Role and Objectives of Control for Wind Turbines. Proceedings of the Institution of Electrical Engineers Pt C, 138, 135-148.

LEITHEAD,W.E., DE LA SALLE, S.A., REARDON, D., 1992, Classical Control of Active Pitch Regulation of Constant Speed HAWTs. International Journal of Control, 55, 845-876.

LEITHEAD, W.E., ROGERS, M.C.M., 1993,A Comparison of the Performance of Constant Speed HAWT's. Renewable Energy - Clean Power 2001, London, IEE Conference Publication No. 385.

MORSE, A.S., 1993, Supervisory Control of Families of Linear Set-Point Controllers. Proceedings of the American Control Conference, San Antonio, 1055-1059.

MORSE, A.S., MAYNE, D.Q., 1992, Application of Hysteresis Switching in Parameter Adaptive Control. IEEE Transactions on Automatic Control, 37, 1343-1354.

PELETIES, P., DECARLO, R., 1993, Asymptotic Stability of 2-Switched Systems Based on Lyapunov Functions. Proceedings of the American Control Conference, San Francisco.

PETTIT, N.B.O.L, WELLSTEAD, P.E., 1994, A Graphical Analysis Method for Piecewise Linear Systems. Proceedings of the 33rd Conference on Decision and Control, Florida.

RICHALET, J. 1993, Industrial Applications of Model Based Predictive Control. Automatica, 29, 12511274. 
ROGERS, M.C.M., LEITHEAD, W.E., 1994, The Dependence of Control Systems Performance on the Wind Turbine Configuration. Report prepared for AEA Technology, University of Strathclyde.

ROGERS, M.C.M., LEITHEAD, W.E., 1993, Relationship of the Controllability of Power/Torque Fluctuations in the Drive-Train to the Wind Turbine Configuration. Proceedings of the 15th British Wind Energy Association Conference, York.

SHEVITZ, D., PADEN, B., 1994, Lyapunov Stability Theory of Nonsmooth Systems. IEEE Transactions on Automatic Control, 39, 1910-1914.

WREDENHAGEN, G., BELANGER, P., 1994, Piece-wise Linear LQ Control for Systems with Input Constraints. Automatica, 30, 403-416.

ZHANG,J.F., 1993, General Lemmas for Stability Analysis of Linear Continuous-Time Systems with Slowly Time-Varying Parameters. International Journal of Control, 58, 1437-1444. 
Figure 1 Linearised control model.

$\mathrm{dQ} / \mathrm{dp}$ is sensitivity of aerodynamic torque, $\mathrm{Q}$, to changes in pitch, $\mathrm{p}$. $\mathrm{dQ} / \mathrm{dV}$ is sensitivity of aerodynamic torque, $\mathrm{Q}$, to wind speed, $\mathrm{V}$.

Figure 2 - Predicted variance of power output vs open-loop cross-over frequency and wind speed (Leithead \& Rogers 1993).

Figure 3 Bode plot of open-loop transfer function with $12 \mathrm{~m} / \mathrm{s}$ member of family of controllers.

Figure 4 Controller structure.

Figure 5 Realisation of inner block of nonlinear controller.

Figure 6 Nonlinear and switched controller open-loop cross-over frequencies

Figure 7 Arrangement used to apply Small Gain theorem to switched linear controller.

Figure 8 Plot of magnitude of $\left(\mathrm{G}_{2}-\mathrm{G}_{1}\right) /\left(1+\mathrm{G}_{1}\right)$ for switched system.

Figure 9 Realisation of switched controller.

Figure 10 Probability density function of power at $24 \mathrm{~m} / \mathrm{s}, 20 \%$ turbulence intensity.

Figure 11 Three standard deviation lines for fits to power maxima.

Figure 12 Pitch acceleration standard deviation. 


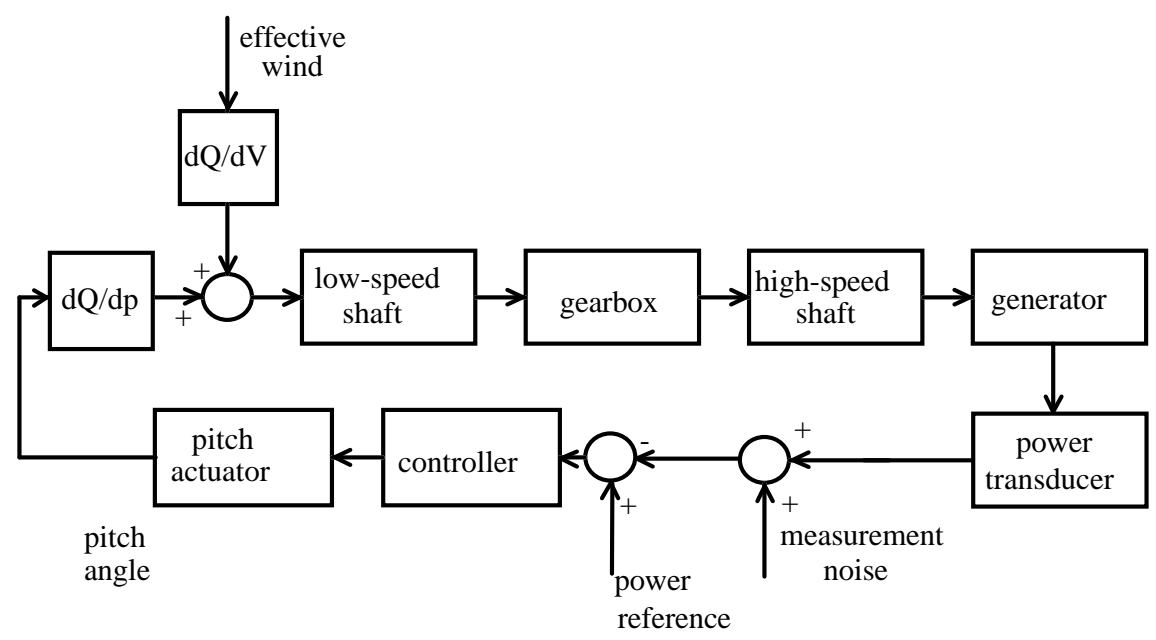


Wind speed $(\mathrm{m} / \mathrm{s})$

$$
\square-12 \mathrm{~m} / \mathrm{s} \longrightarrow-\prec 16 \mathrm{~m} / \mathrm{s} \longrightarrow 23 \mathrm{~m} / \mathrm{s}
$$

$\log \left(\right.$ variance of power) $\left(\log \left(k W^{\wedge} 2\right)\right)$

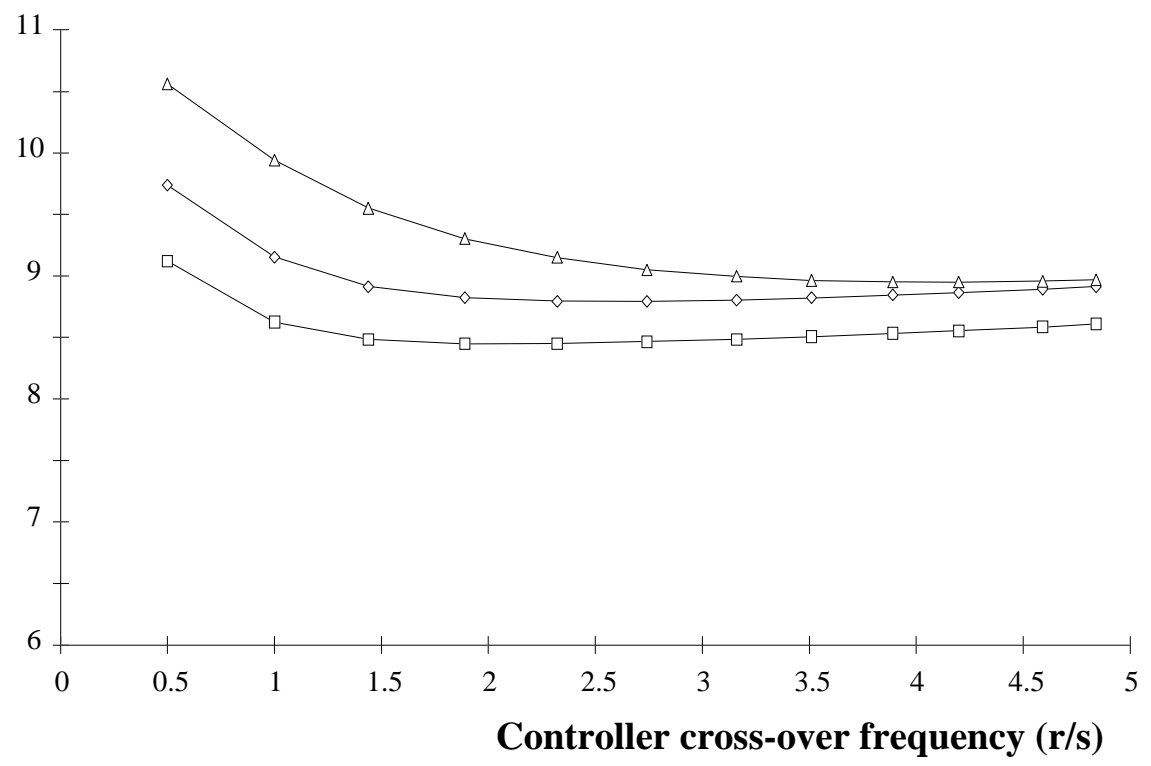



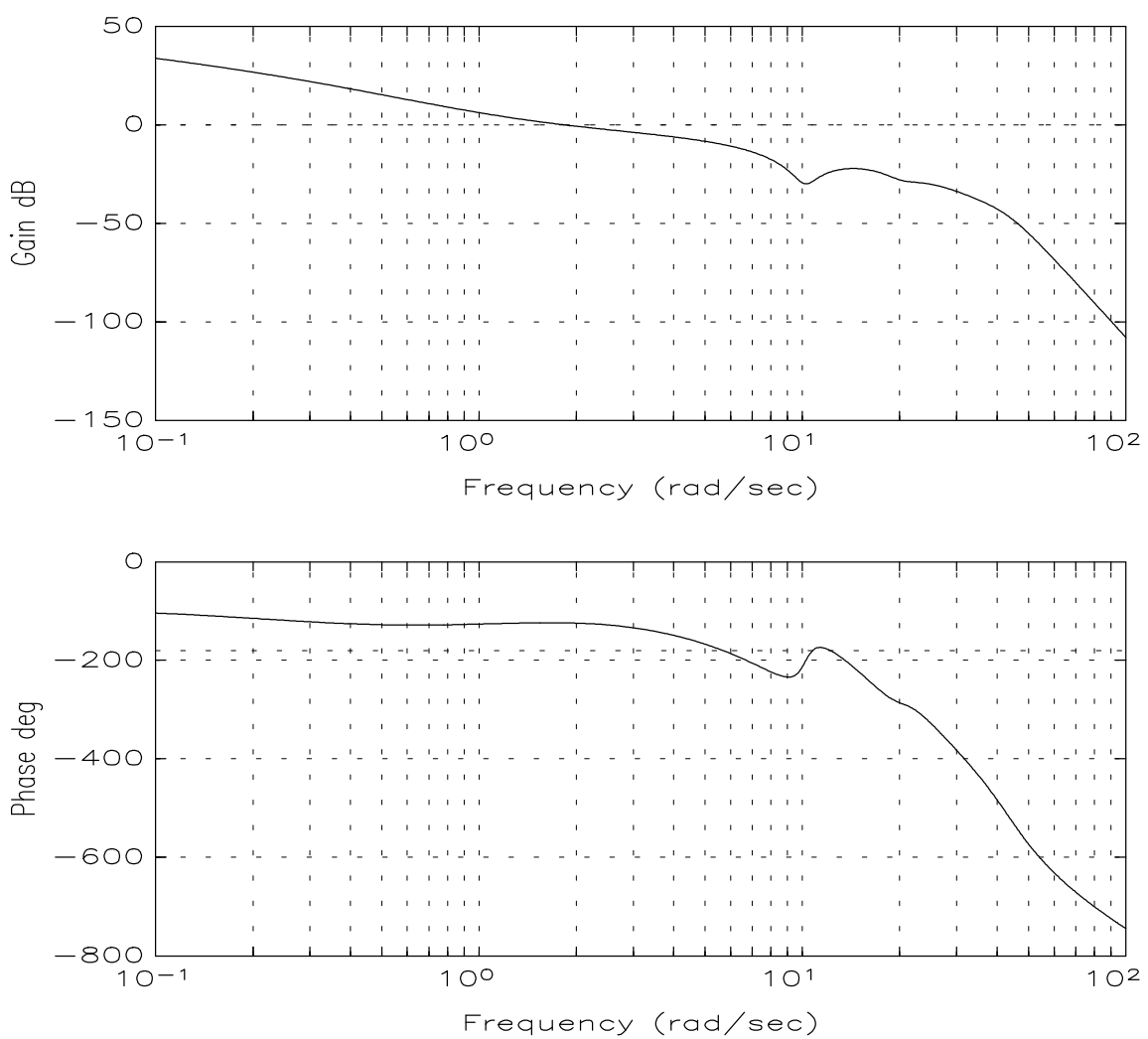


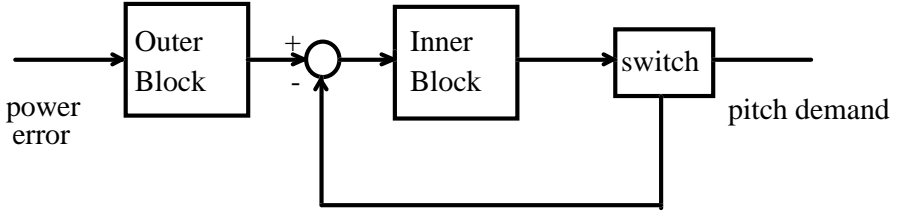




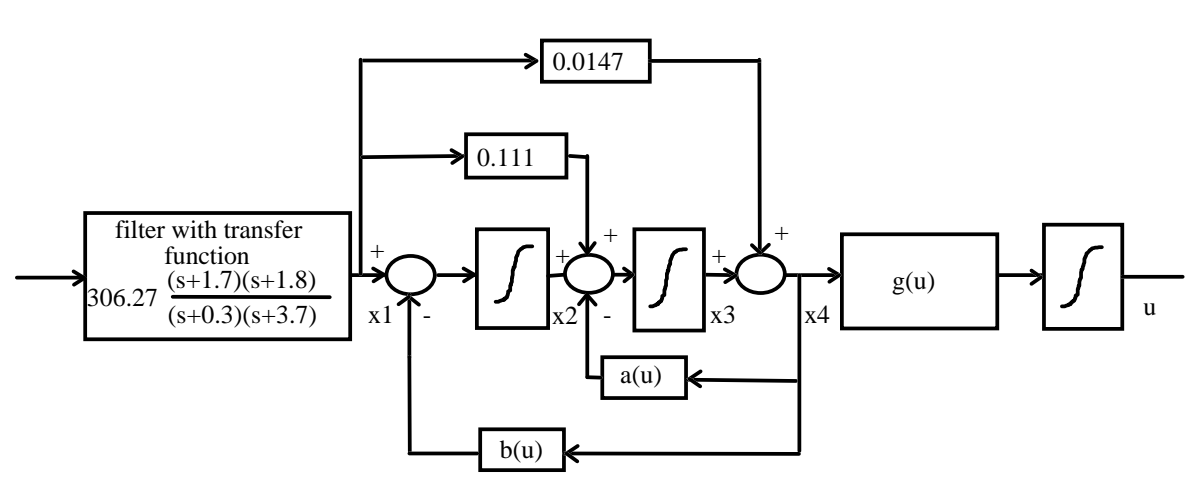


open-loop cross-over frequency $(\mathrm{r} / \mathrm{s})$

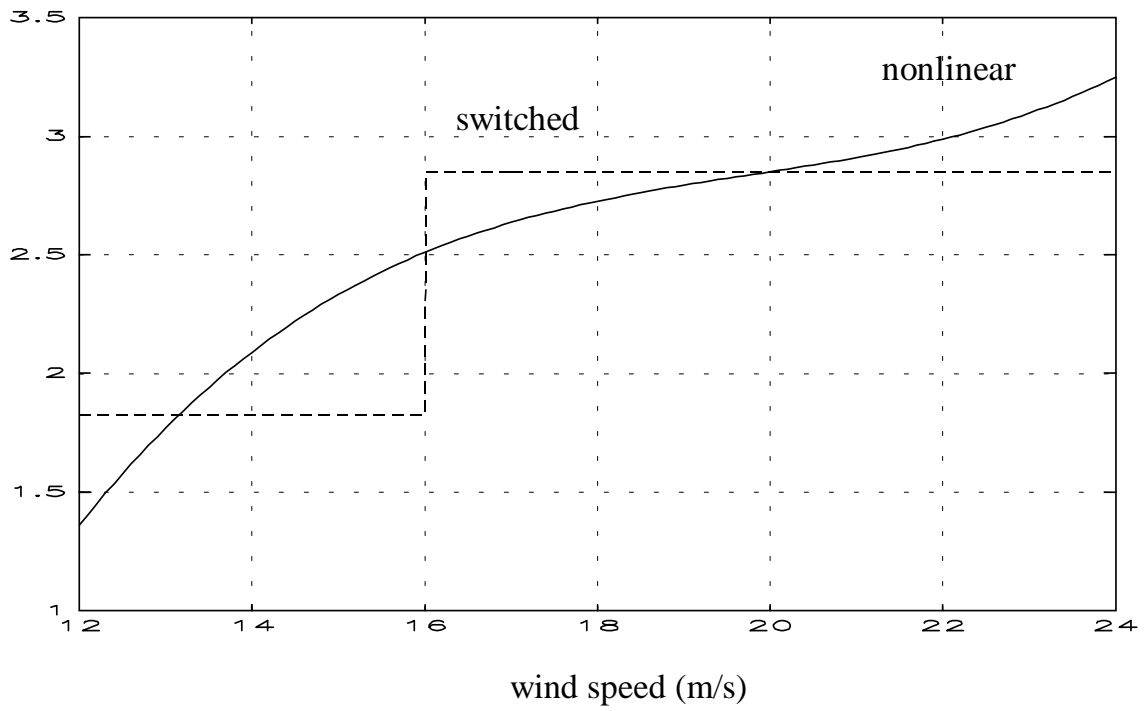




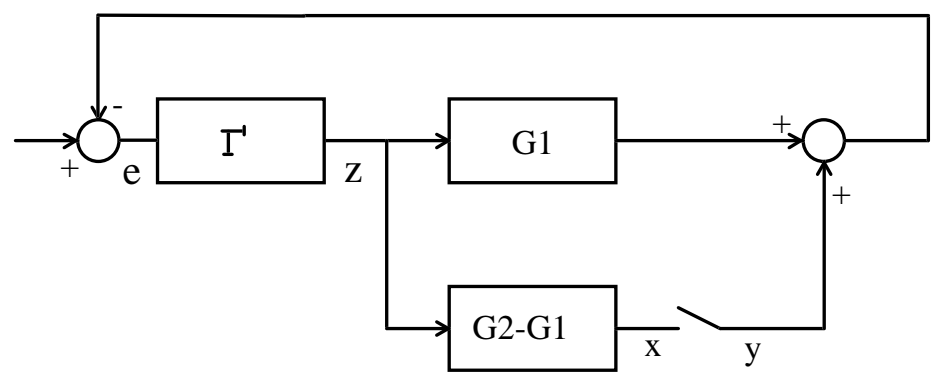




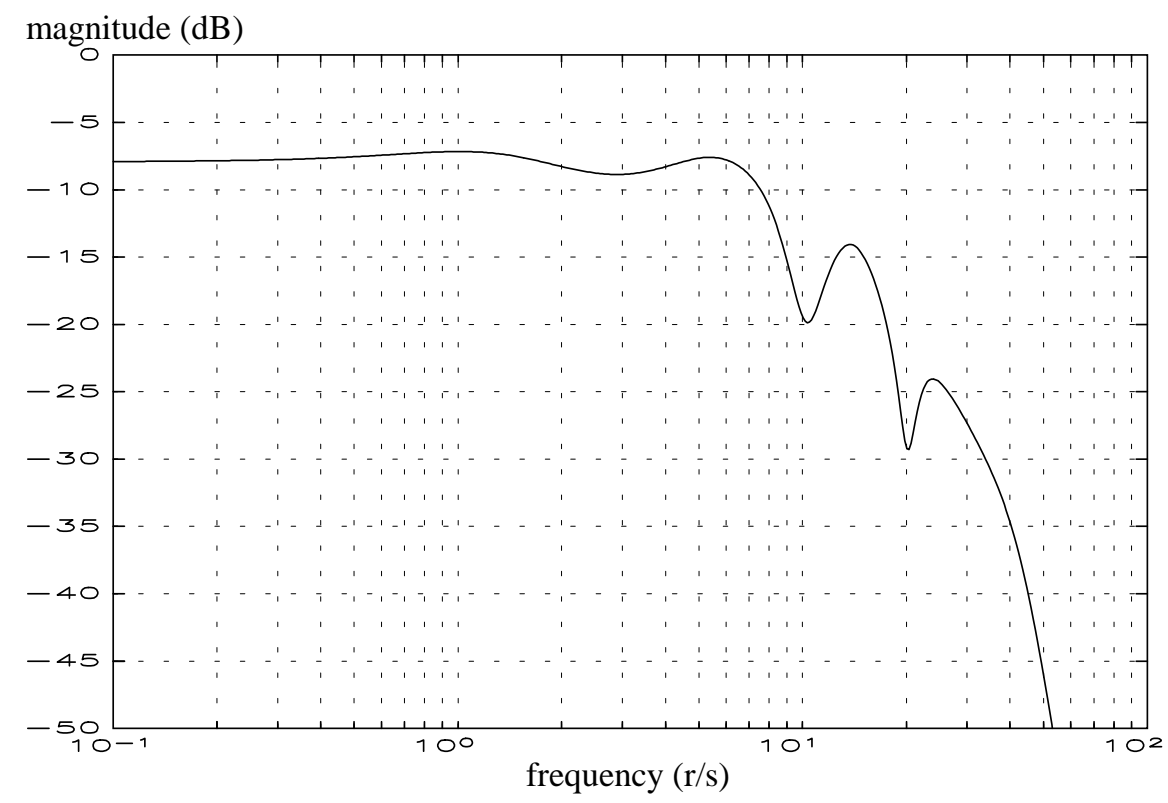




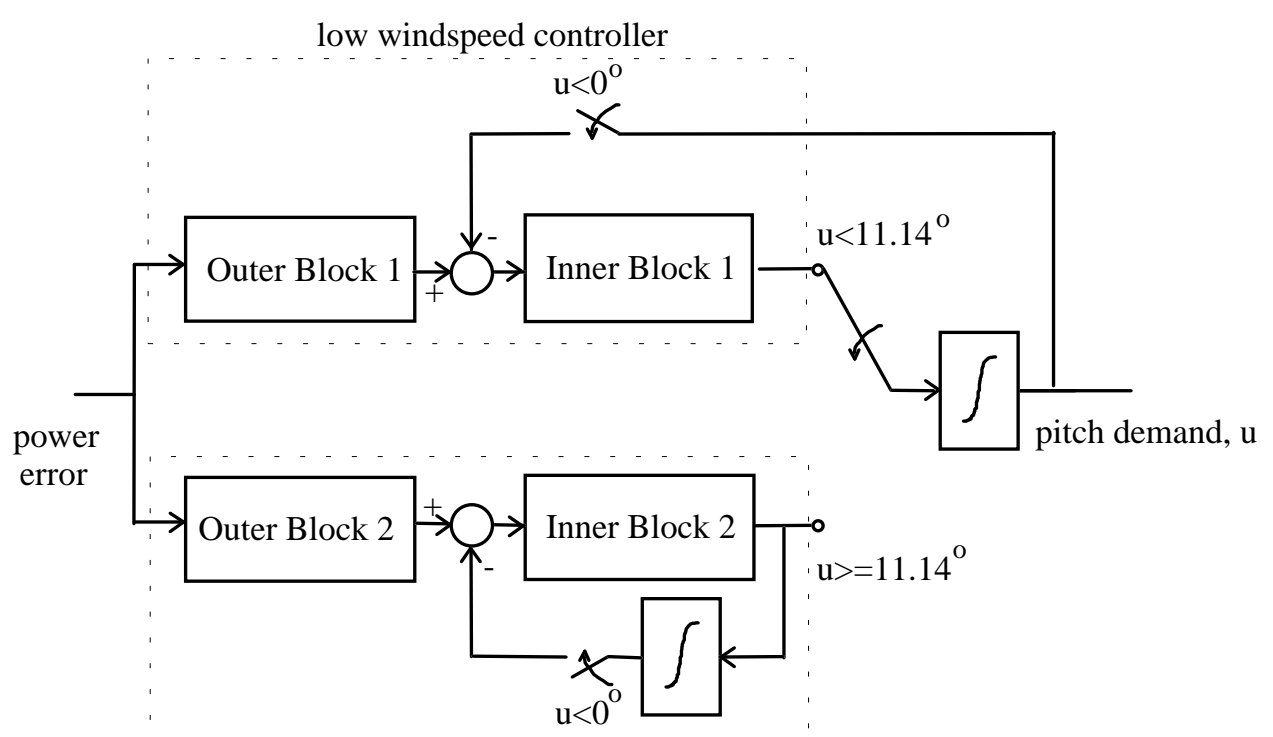

high windspeed controller 


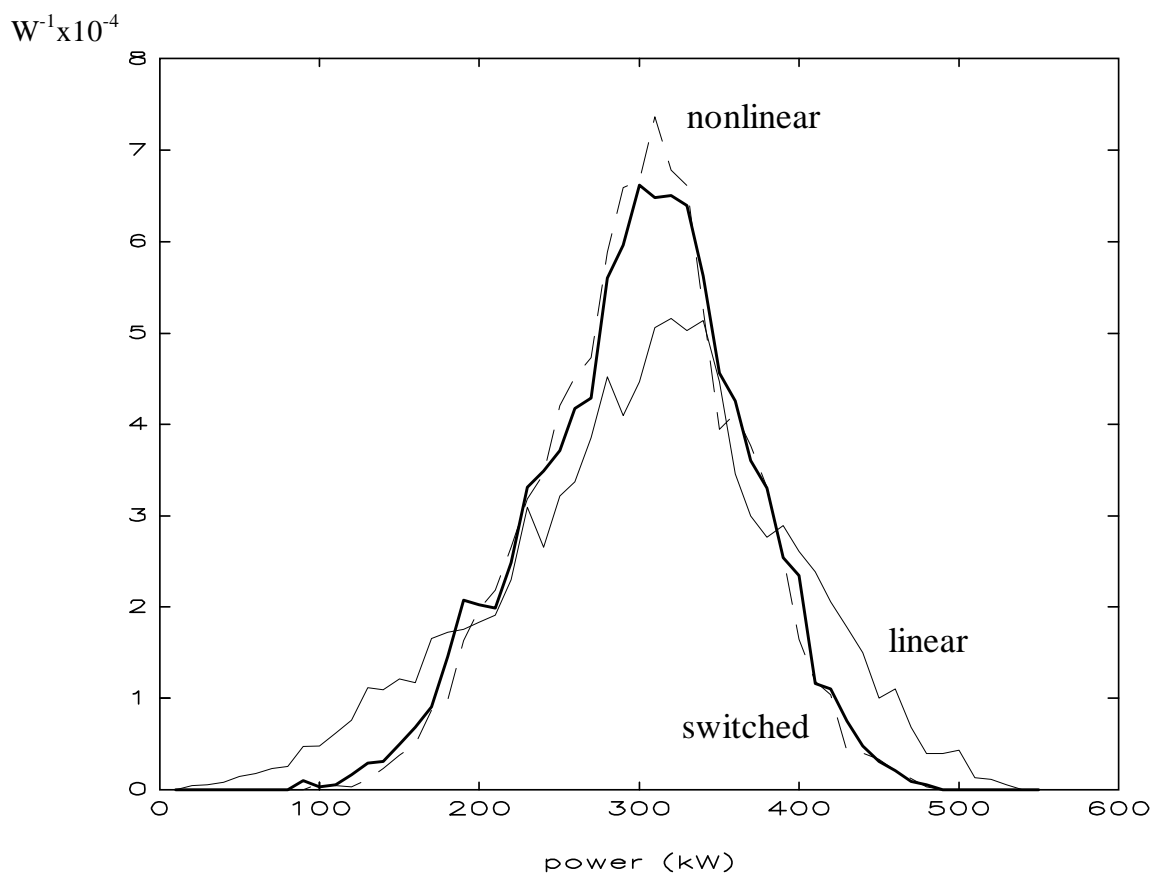




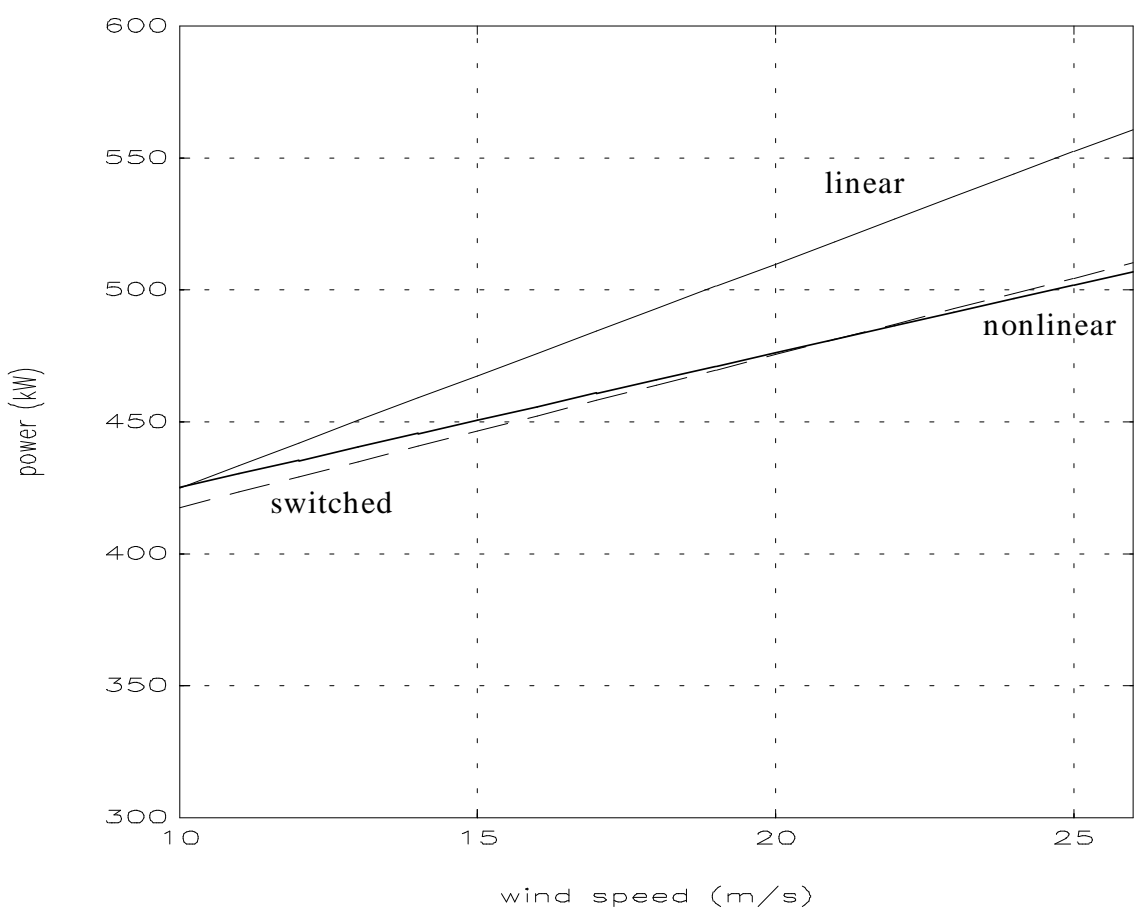




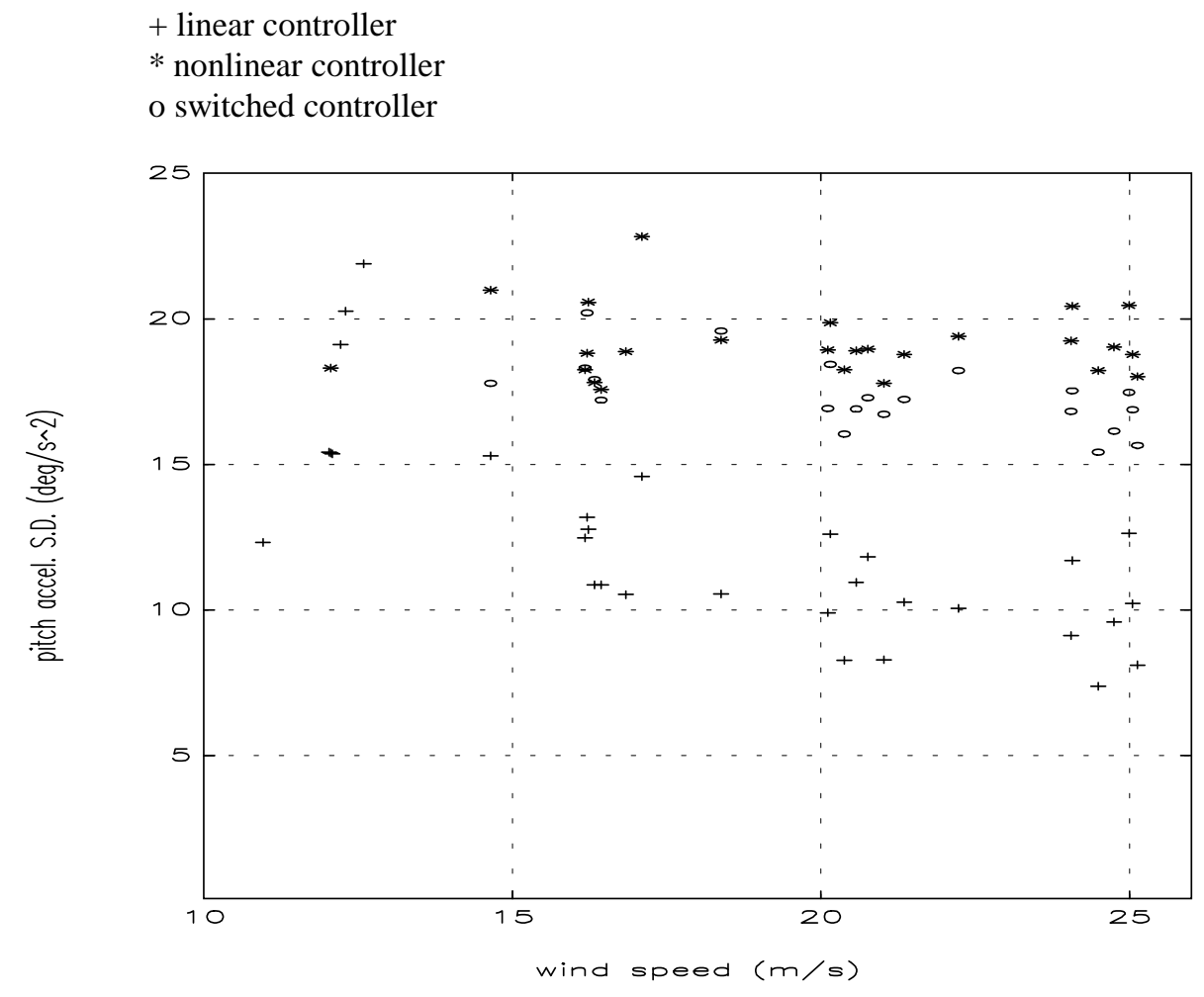

